\title{
A Study on Wang Rongpei's Translation of the Complete Works of Tao Yuanming*
}

\author{
Juju Wang \\ School of Foreign Studies \\ QiLu Normal University \\ Jinan, China 250013
}

\begin{abstract}
It is widely acknowledged that Chinese ancient poems are the one beyond comprehension and translation. Tao Yuanming is best known to be the idyllist whose poems are famous for the natural and simple. Wang's translational technique used in Tao's poems achieved extraordinary artistic effect. This paper analyzes his translation from the perspective of language, literature and culture, which contributes to convey the images, concepts and amplifications of the original one.
\end{abstract}

Keywords-Wang Rongpei; The Complete Works of Tao Yuanming; language; literary; culture

\section{INTRODUCTION}

Wang Rongpei is a firm-minded and practical translator who has brought many Chinese classics like the Book of Songs, the Book of Changes, Zhuang Zi, the Peony Pavilion, the Complete Dramatic Works of Tang Xianzu etc. to the rest of the world. The Complete Works of Tao Yuanming is the first integral translation of Tao's poetries, and at the same time Wang Rongpei's monograph the Comparative Study of Tao Yuanming Poetries' English Versions was published. Wang's indefatigable and realistic spirit behind his extraordinary translation is explicitly reflected to us. In order to find the perfect translation, Wang tried to learn Tao's experience and personality as well as his thoughts and writing styles. Studying Tao's related books and articles, visiting Tao's hometown and his descendant, collecting more than 20 English versions of Tao's poetries, all these works took him 2 year's time.

The Complete Works of Tao Yuanming consists of 122 Tao's extant poetries, which are all 5-word ancient poems except of 9 4-word poems. Although Wang seldom discusses translational theory, at the preface of the Complete Works of Tao Yuanming, he cites that my translational principle always be conveying the spirit and meaning which is boiled down from his years of translating practice. The beauty from image, conception and implication could be analyzed from the perspective of language, literature and culture. From these 3 levels, this paper explores Wang's translational methods and techniques which contribute the spectacular artistic effects.

*A Phased achievements of the "Study on Translating Teaching Strategies Guided by Translational Stratigies " of 2017 Young Teachers' Scientific Research Program of Qi Lu Normal University (2017JX2302).

\section{WANG'S TRANSLATIONAL TECHNIQUES IN LANGUAGE PROCESSING}

Characters in Chinese language originated from the hieroglyphic which made from painting, while gradually the painting properties are decreased as its symbol nature increased. This word-formation system has many limitations, as many concrete and abstract things cannot be painted. Therefore, on the basis of hieroglyphic, ideographic method was developed like semasiography, self-explanatory and echoism, all of which were made by combining, adding or reducing symbolic signs based on existing hieroglyphic. Germany philosopher G.W. von Leibriz once described Chinese characters as speaking with visual images (Yao Xiaoping, 2006). The image aesthetic in the original text would be lost completely when translated into letter words.

What's more, poem's tones-one of the element of Chinese characters- would be lost in the English translating process. As a tonal language, the four tones in Chinese language produce the vocal Varity and form a musical feature. Meanwhile, most of the Chinese characters are monosyllable, while English is intonation language and multi-syllable with stress in their words. Because of the phonetic features, the rhythm of the Chinese poems has level or oblique tone rules, but English poems' rhythm has soft and stress pattern. Therefore, the language game with phonetic features is hard to be translated between English and Chinese languages.

Wang Rongpei constructed the poem translational language revolving with English grammar which enables to lead the target readers to appreciate smoothly and successfully. British translator Peter Bush compared literary translation as struggle consisting of two problems: understanding the original text and creating the translation (Sun Yingchun, 2005). Tao Yuanming applied the conversational language to write poetry, which can display his unique aesthetic standard. Wang's translational skill is quite dialectical with loyalty to the original text first and expressing with standard target language. This kind of authentic translation not only let the readers feel regret about the loss of Chinese ancient poems' literary grace and aesthetic image.

Wang's translation of the poem Back to Country Life (3) is a good case in point. 
When I plant beans at the foot of Southern Hill,

Bean shoots are few but rank grass grows at will.

I rise at early dawn to week and prune

Till, hoe on shoulder, I return with the moon.

As the path is narrow, grass and bushes tall,

The evening dew will soak my dress and all.

It's nothing when my dress gets wet with dew,

As long as my desires indeed come true.

The original poem is 5-word ancient poem, and in Wang's English version all the sentences are pentameters. Tao's Chinese poem is rhymed in the even numbered lines while Wang's version is rhymed couplets and rhymed pattern is aabbccdd. The first line is translated into when I plant beans at the food of Southern Hill, Bean shoots are few but rank grass grows at will. The proper noun Southern Hill refers to Lushan Mountain south of Tao's home, which is capitalized on purpose by Wang. The word rank is a derogatory term meaning weed infestation, excessive growth. It is the understanding of Tao's intention that Wang chose rank grass rather than flourishing weeds, weeds grow thick, weeds flourished or weed abound which are positive words. The word "few" is better than "sparse" or "rare" with both negative meaning and the concept of number. The absolute structure "hoe on shoulder" could show us the vivid image of working in the field, and also keep the consistent rhythmic pattern. It is acknowledged that, when it comes to narration, the poet in ancient China often used the first person which was seldom appeared in the lyrics. In English poem the situation of hiding person and omitting the subject is hard to find. In Wang's translation, "I" referring to the writer is supplemented naturally.

Wang's profound English competence makes it possible to translate Chinese ancient poems naturally. In his translation, from elaborate using of synonyms, antonyms, elliptical constructions, rhythm's choosing, to rhyme steps' selection all can reveal Wang's accurate understanding and excellent skills of using different languages.

\section{WANG'S TRANSLATIONAL TECHNIQUES IN LITERARY PROCESSING}

Chinese literature is unique like Chinese characters, which referred to all the written books in ancient times. Literature is the term for the arts which reflects the social life, expresses the emotions with written language in now days. The references of oriental and occidental literature are quite different, and poetry which represents the highest state of linguistic art is not the exception. The remarkable essence of Chinese ancient poetry is not only shown in the image of the character and tones, but also in the artistic conception between the lines. Wang's not satisfied with the language level, but intends to lead the target readers appreciate the artistic concept beyond the lyrics.

Wang believes the most important feature of Tao's poems is "natural" which represents his worship of the nature. As Wang once pointed out that Tao wrote whatever in his mind and in the way he wanted without any mannerism. Tao described the most common things in everyone's daily life with average people's words. Reproaching My Son is an good example, which like the normal conversation with our neighborhood or friends who are disappointed with their sons. Unlike other ancient obscure poems which contain unpronounceable words and mysterious principles, this poem depicts a kind but frustrated father. The natural and plain state is perfectly illustrated in Wang's translation.

My hair at both my temples have turned grey;

My skin and muscles weaken day by day.

Although I have five sons in my own right,

None of them would like to read and write.

Although Ashu is sixteen years of age,

$\mathrm{He}$ is more lazy than the average.

Although Axuan will soon come to fifteen,

Found of pens and books he's never been.

Young and Duan, thirteen years old by now,

Cannot tell six from seven anyhow.

What my nine-year-old Atong now cares,

Is no more than to pick the nuts and pears.

If this has been the destined fate of mine,

I'd better sit and drain the cup of wine.

The natural and simple style in the original poem is retained in the English version without any big, abstract or unfamiliar words. This can be more specific compared with other translation like the following one. My eldest son already is twice eight, For laziness none can be his compeers. The usage of "twice eight" is not common and natural, while the "laziness" is abstract term and compeers the obscure one. Tao's poem and Wang's translation prove one truth that is the truth is simple.

\section{WANG'S TRANSLATIONAL TECHNIQUES IN CULTURAL PROCESSING}

Duo to the visualized language as well as the toned feature, a few characters can combine a poem. Chinese cultural is a kind of poetic one compared even contrary with the logic English culture. The language and literature are all contained in culture with language as its basic carrier. As far as the perspective of Chinese person's emphasize on intuition, Chinese are good at radial thinking like metaphor, symbolization, and implication. Ancient Chinese poem is the unique and concise form of the culture which may be lost completely in the process of translation. Some cultural implications in Chinese ancient poems seem embarrassed even awkward in the English linguistic environment with English readers' cultural imagination.

Many literary and cultural implications could be found in Tao's poems, most of which related with natural sceneries 
like wind, cloud, birds and chrysanthemum. The most frequently appeared cultural image is "wind" which totals 55 times. If physically moved into the English environment, even with the accurate and logic language, the wind's cultural concept in the poem's background and its literary image with the distinctive feature of Tao's poem cannot fully reflected. Wang simply literally translated it as wind without any interpretations in order to intrigue the target readers inspiring, reflecting. It is Chinese ancient poetry's traditional distinctions of meaning beyond the lines that makes this translational skill possible, feasible and necessary in its translation. Undoubtedly, the isolated poetry feature in Chinese cultural environment makes Wang's translational strategy appropriate and proper.

Tao Yuanming lived in the Eastern Jin Dynasty (317-420) characterizing with national secession and political unrest. At that time, Confucius thoughts still exited and upholding standard behavior and doing right things according to fixed rules. Taoist ideas were prevailing encouraging people to confront the nature and universe directly without social restraints. At the same time, Buddhist thought became popular too advocating disciplining oneself now and looking forward to the future after life. Tao's pursuit of serving the country and the people, focusing on personal cultivation was influenced with Confucius thoughts, while his respecting nature and pursuing detachment were the reflection of Taoism and Buddhism. All the history background and cultural connotation cannot be appreciated and properly translated by the sinologists in the west.

\section{CONCLUSION}

As cited in the prelude of The Complete Works of Tao Yuanming, Wang Rongpei pointed out that my translational principle is conveying the spirit and meaning. Tao's peoms are rhythmic, so I adopted rhyme translation which might lead damaging the meaning for the sake of rhythms. Through the significance of poetry translation is expressing the image not every details. Conveying spirit refers to original spirit in the poem like form, tone, image, and figures of speech. Conveying the meaning refers to the deep and surface meaning. All the efforts could be seen from the linguistic, literary and cultural levels.

Tao's translation by Wang is the first complete one in China, and many of which has been translated before. To a certain extent, Wang's translation is retranslation which is more difficult with the aim at transcending previous ones. Before translation, Wang collected various versions for comparison, visited Tao's hometown and his descendents, and studied all articles and papers related with Tao. Without these efforts, Wang's version could not achieve spectacular effects both in art and academy.

\section{REFERENCES}

[1] Fang Chong, The Selected Translation of Tao Yuanming's Poems [M]. Shanghai: Shanghai Foreign Language Education Press, 1984

[2] Hou Xiangqun, Appreciation of Wang Rongpei's New Translation [J]. Foreign Language and Their Teahcing, 2001:52-55
[3] Wang Rongpei, the Complete Works of Tao Yuanming [M]. Changsha: Hunan People's Press, 2005

[4] Wang Rongpei, the Comparative Study of Tao Yuanming Poetries' English Versions [M]. Changsha: Hunan People's Press, 2005

[5] Sun Yingchun, Studies on Zhang Guruo's Translaitonal Arts [M]. China Translation and Publishing Corporation, 2005

[6] Yao Xiaoping, Ten Speeches on Language and Culture [M].Foreign Language Teaching and Research Press. 2006 